\title{
La teoría autónoma del sentido de Katz y la determinación de la referencia de términos de clases naturales
}

\author{
José Alejandro Mosqueda Esparza \\ Facultad de Filosofía y Letras / Instituto de Investigaciones Filosóficas \\ Universidad Nacional Autónoma de México \\ mosquedaesparza.josealejandro@gmail.com
}

\section{Introducción}

La teoría autónoma del sentido es una alternativa que postula Katz frente al intensionalismo fregeano. Katz argumenta que el intensionalismo fregeano padece del problema de la restricción demasiado débil, y esto lo lleva a la indeterminación del sentido. Un paso clave para librar tal problema es, según Katz, independizar la teoría del sentido de la teoría de la referencia. Sin embargo, a pesar de que con ello se logra rechazar el intensionalismo fregeano y así librar la indeterminación del sentido, la propuesta de Katz adquiere nuevos problemas para determinar la referencia de términos de clases naturales. Cohen señala que la única alternativa de Katz para explicar con éxito cómo se determina la referencia de estos términos es aceptar que las definiciones reales hacen todo el trabajo. Pero aceptar esto nos lleva a concluir que el sentido de un término $t$ no es necesario para caer en la exten- 
sión de $t$. Katz no puede aceptar tal conclusión, puesto que su nuevo intensionalismo sostiene que el sentido es necesario, pero no suficiente para la referencia. Por lo tanto, parece que la estrategia de Katz fracasa al intentar determinar la referencia de términos de clases naturales.

Si bien la noción del sentido de Katz permite hacer uso de evidencia intensional y de este modo evita el problema de la indeterminación del sentido, es interesante ver si tal noción proporciona condiciones necesarias para determinar la referencia de un término de clase natural. A diferencia de la tesis extensionalista para la cual el sentido no es ni necesario ni suficiente para la referencia, Katz defiende un intensionalismo no fregeano que sostiene que las expresiones tienen sentido sobre y por encima de su referencia, y además que los sentidos no determinan la referencia (ver Katz, 2004: 7). El objetivo de este ensayo es analizar si la afirmación de Katz de que el sentido de un término $t$ es necesario para caer en la extensión de $t$ puede sostenerse.

Noción fregeana del sentido: la reducción de la teoría del sentido a la teoría de la referencia

Katz cree conveniente distinguir entre un Frege real y uno mítico. Frege sostuvo «que las expresiones del lenguaje tienen sentido sobre y por encima de su referencia (intensionalismo); pero también sostuvo que el sentido y la analiticidad son fundamentalmente nociones referenciales (intensionalismo fregeano)» (Katz, 2004: 3). De acuerdo con Katz, al Frege real no le interesa la semántica de los lenguajes naturales y mantiene un proyecto logicista de fundamentación de las matemáticas. Pero también se ha creado un Frege mítico a 
partir de la interpretación de Sobre sentido y referencia, la cual se interesa por introducir la noción de sentido en el lenguaje natural y con ello llevar más allá los propósitos logicistas de la semántica de Frege.

En Sobre sentido y referencia, Frege considera dos oraciones:

(1) Héspero es Héspero $[\mathrm{A}=\mathrm{A}]$.

(2) Héspero es Fósforo $[\mathrm{A}=\mathrm{B}]$.

Suponiendo que (1) y (2) son verdaderas, ¿en qué reside la diferencia entre ambos enunciados? Lo primero que hay que notar es que podemos conocer a priori la oración (1), mientras que (2) se conoce generalmente a posteriori. Entonces para conocer (2) necesitamos extender nuestro conocimiento más allá del conocimiento que tenemos del signo «Héspero»y del signo «Fósforo».

¿Cómo explicar la diferencia en valor cognitivo entre enunciados del tipo «Héspero es Fósforo»? La respuesta de Frege es que si es el caso que $« \mathrm{~A}=\mathrm{B}$ » es verdadero, entonces lo sería porque tanto «Héspero» como «Fósforo» refieren a una y la misma cosa. A cada signo le corresponde un sentido determinado y a su vez a cada sentido determinado le corresponde una referencia determinada. Sin embargo, a una referencia no le corresponde sólo un signo y por ende tampoco le pertenece un solo sentido:

Así pues, resulta natural pensar que con un signo (nombre, unión de palabras, signos escritos) está unido además de lo designado, lo que se podría llamar la referencia del signo, lo que me gustaría llamar el sentido del signo, donde está contenido el modo de presentación. De acuerdo con esto, en nuestro ejemplo [...] la 
referencia de «el lucero de la mañana» y el «lucero de la tarde» es la misma, pero no el sentido (Frege, 1998: 85).

Al signo «Héspero» le corresponde el sentido «el lucero de la tarde», al signo «Fósforo», el sentido «el lucero de la mañana», y el referente de ambos sentidos es Venus. De esta manera lo expresado por (1) y (2) quedaría como sigue:

(1a) El lucero de la tarde es el lucero de la tarde.

(2a) El lucero de la tarde es el lucero de la mañana.

Para conocer que el lucero de la tarde es el lucero de la mañana necesitamos ampliar nuestro conocimiento.

La referencia de un signo es un objeto, mientras que el sentido de un signo es el modo de presentación (la manera de designar al objeto) del referente que no es parte o no es un modo de una mente individual, sino que puede ser propiedad común de muchos (ver Frege, 1998: 87-8). Lo que según Katz hay que notar de esta noción del sentido es que la distinción entre sentido y referencia se lleva a cabo dentro de la teoría de la referencia. En una noción del sentido basada en Frege el «sentido es el aspecto de las expresiones, en virtud del cual se refieren a ciertos objetos en el dominio del lenguaje; los referentes son cosas en el dominio al que se refieren las expresiones, en virtud de su sentido. El sentido y la referencia son, por lo tanto, el determinador y lo determinado, respecto de la relación de referir» (Katz, 2004: 13). El sentido se entiende únicamente como el instrumento para hacer referencia. El problema que plantea Katz a partir de la noción fregeana del sentido es si ésta nos permite decidir cuál es el sentido correcto de un símbolo u oración: ¿son las restricciones que impone la noción fregeana de sentido lo su- 
ficientemente fuertes como para individuar los sentidos de las expresiones?

\section{El problema de la restricción demasiado débil y el argumento de la indeterminación del sentido}

Para Katz las restricciones que impone la noción fregeana de sentido no son lo suficientemente fuertes como para individuar los sentidos de las expresiones, es decir, la definición del sentido no nos dice lo suficiente acerca del sentido como para permitirnos determinar los sentidos correctos de las expresiones. A este problema lo llama «la restricción demasiado débil» (ver 2004: 24-30). Se ha mencionado en el apartado anterior que Frege explica el sentido en términos de la función que adopta dentro de la teoría de la referencia:

Esto hace que la individuación de los sentidos sea, como la individuación de las herramientas, una cuestión de función. Una vez que los intensionalistas definen el sentido funcionalmente, se comprometen a decir que cualquier cosa que satisfaga la descripción de la tarea «determinador de la referencia del término $t$ » cuenta como el sentido de $t$ (Katz, 2004: 25).

Y lo que es peor, al adoptar este compromiso los intensionalistas fregeanos limitan a evidencia extensional los criterios para individuar los sentidos de una expresión.

Así, la noción fregeana del sentido basa su explicación sólo en consideraciones referenciales. La definición fregeana del sentido se explica en términos del papel que desempeña dentro de la teoría de la referencia, lo que hace que la individuación de los sentidos sea una cuestión de función. 
Entonces lo que individua a los sentidos es el papel que desempeñan — determinar la referencia de un término-, y de acuerdo con esto, el sentido de dos signos diferentes debe tomarse como el mismo, en virtud del hecho de que determinan el mismo referente. Katz da un ejemplo en el que resulta problemático pensar que el sentido pueda tomarse como el mismo: de acuerdo con el intensionalismo fregeano, el sentido de la expresión «dos» y el sentido de la expresión «el número primo par» deben tomarse como el mismo, en virtud del hecho de que determinan al mismo referente en todo mundo posible. Sin embargo, estas expresiones no tienen el mismo sentido (pues es necesario probar que el dos es el número primo par). La diferencia entre los sentidos de estas expresiones es que tienen diferentes propiedades intensionales, por ejemplo, la propiedad de ser el único número que es par y primo es parte del sentido de «el número primo par» pero no es parte del sentido de «dos». No obstante,

desde el punto de vista del intensionalismo fregeano, donde tales diferencias no son relevantes, podemos decir que el sentido de «dos» es: el número primo par; y también podemos decir, por las mismas razones, que el sentido de «dos» es: el sucesor de uno, la raíz cuadrada de cuatro, la diferencia entre quince y diecisiete, $\mathrm{o}$ cualquier otro que sea necesariamente coextensivo con el sentido de «dos» (2004: 25).

Por lo tanto, en el intensionalismo fregeano

no hay manera de elegir el sentido correcto de una expresión entre muchos sentidos variados, todos los cuales necesariamente determinan el mismo referente, porque cualquier sentido que lle- 
ve a cabo la tarea de determinar al referente de la expresión, es tan bueno como cualquier otro que también haga la tarea (2004:26).

Y esto es una consecuencia de la definición fregeana del sentido en la que se reduce la teoría del sentido a la teoría de la referencia. Esta reducción hace que sólo los criterios extensionales restrinjan las elecciones entre las hipótesis semánticas, y además elimina los criterios intensionales que podrían determinar que una hipótesis sea la correcta. Con todo lo anterior, es posible concluir que hay simetría en la evidencia relevante para elegir entre hipótesis incompatibles sobre el sentido de un término y, por lo tanto, es indeterminado cuál es el sentido correcto del término.

A grandes rasgos, el problema con la noción fregeana del sentido es que no hay un criterio que nos permita elegir cuál es el sentido correcto de un término. Para cada término existen varios sentidos que cumplen con el criterio intensionalista fregeano, es decir, hay muchos sentidos que determinar el mismo referente. $Y$ ya que ese es el único criterio - consecuencia de reducir la teoría del sentido a la teoría de la referencia-, no hay nada que impida la simetría en la evidencia relevante para elegir entre hipótesis incompatibles sobre el sentido de un término. Por lo tanto, es indeterminado cuál es el sentido de un término. Esto supone lo siguiente:

1) La evidencia relevante para individuar el sentido de un término $t$ es que el sentido de $t$ lleve a cabo la tarea de determinar al referente del término $t$ (Premisa fregeana).

2) Si (1) es correcta, entonces hay simetría en la evidencia para hipótesis incompatibles sobre cuál es el sentido de $t$. 
3) Si hay simetría en la evidencia para hipótesis incompatibles sobre cuál es el sentido de $t$, entonces es indeterminado cuál es el sentido de un término.

4) Es indeterminado cuál es el sentido de un término.

La tesis fregeana de que el sentido de un término determina su referencia apoya la verdad de 1). Por otro lado, 2) nos dice que es posible que exista la misma evidencia a favor de la hipótesis de que el sentido $S 1$ es el sentido correcto de un término $t$, que la evidencia que existe a favor de la hipótesis de que un sentido $S 2$ es el sentido correcto de $t$; a pesar de que ambas hipótesis sean incompatibles entre sí. Si hay tal simetría en la evidencia para hipótesis incompatibles, entonces es indeterminado cuál es el sentido de un término.

La definición fregeana del sentido crea el problema de la indeterminación, puesto que la explicación de la noción del sentido se basa sólo en consideraciones referenciales. Katz confía en que con una noción autónoma del sentido sea posible reforzar las restricciones sobre la determinación del sentido, permitiendo que haya consideraciones no referenciales, es decir, evidencia intensional (ver 2004: 29).

La noción autónoma del sentido: el rechazo

al intensionalismo fregeano

Para evitar la conclusión del argumento anterior - la indeterminación del sentido- es suficiente con rechazar 1), es decir, Katz necesita negar el intensionalismo fregeano. Se ha adelantado en la introducción de este ensayo que una noción del sentido, como la de Katz, permite hacer uso de evidencia intensional y así evita el problema de la indeterminación del 
sentido; además, que el sentido de un término de clase natural proporciona condiciones necesarias para la pertenencia a la extensión de dicho término. Hay dos pasos principales para el desarrollo de esta tesis: el primero es argumentar que la noción autónoma del sentido de Katz evita la indeterminación del sentido; el segundo es explicar la relación entre la teoría autónoma del sentido y la teoría de la referencia, a partir del rol que juega la teoría autónoma para determinar la referencia de un término. En este apartado se desarrollará el primer paso, y el segundo se tratará en el quinto apartado.

La indeterminación del sentido es causada por el problema de la restricción demasiado débil, que a su vez, es una consecuencia de reducir la teoría del sentido a la teoría de la referencia. Entonces, parece que si se independiza la teoría del sentido de la teoría de la referencia se evita la indeterminación del sentido. Es decir, Katz necesita negar 1) del argumento de la indeterminación de la referencia expuesto al final del apartado anterior:

1) La evidencia relevante para individuar el sentido de un término $t$ es que el sentido de $t$ lleve a cabo la tarea de determinar al referente del término $t$ (Premisa fregeana).

Hay que recordar que para Frege el sentido era el aspecto de las oraciones mediante el cual se refieren a ciertos objetos en el dominio del lenguaje. El sentido de un signo era el modo de presentación del referente o la manera de designar al objeto (ver Frege, 1998: 87-8). En oposición a esta definición fregeana del sentido, Katz ofrece (D): «el sentido es ese aspecto de la estructura gramatical que tienen las oraciones, y que es responsable de sus propiedades y relaciones del sentido» (2004: 17). Con tal definición, el sentido ya no es 
«el determinador»y, por lo tanto, su tarea ya no es determinar al referente de un término. No obstante, de acuerdo con (D), los sentidos aún son determinadores, pero lo que ahora determinan son propiedades y relaciones del sentido: «Las propiedades y relaciones del sentido [...] reflejan la estructura gramatical de las oraciones dentro de las oraciones de un lenguaje. [...] Al decir que son internas a la oración, (D) independiza al sentido de la referencia y hace que la teoría del sentido sea autónoma» (2004: 17). El sentido de una oración ya no es el aspecto que lleva a cabo una función dentro de la teoría de la referencia, sino que ahora es el aspecto de la estructura gramatical que tiene la oración, y que es responsable de las propiedades y relaciones del sentido.

Para el intensionalismo fregeano, el sentido era el aspecto de la oración gracias al cual la oración se refería a ciertos objetos en el dominio del lenguaje. A partir de la independencia de la teoría del sentido, la noción del sentido ya no se limita a ser un aspecto de la manera en que las oraciones se refieren a un objeto. Su papel, ahora independiente de la relación entre el determinador y lo determinado, es reflejar la estructura gramatical de las oraciones y con ello proporciona evidencia intensional. Ya que con (D) se independiza la teoría del sentido y el sentido ahora es el aspecto de la estructura gramatical que tienen las oraciones, entonces la evidencia relevante para individuar el sentido de un término es evidencia intensional. Con esto Katz rechaza 1).

Un punto importante de la independencia de la teoría del sentido, respecto de la teoría de la referencia, es que ahora el sentido nos permite incorporar evidencia intensional para individuar el sentido de un término. Es decir, la noción del sentido de Katz nos dice que el sentido es el elemento de la oración que se encarga de las relaciones y propiedades del sentido 
que mantiene la oración con el lenguaje. Estas propiedades son o bien expresivas del sentido, cuando son simplemente el conteo del número de sentidos que tienen las expresiones; o bien no expresivas, cuando son tipos de estructuras de los sentidos. Se tienen entonces como propiedades y relaciones expresivas: tener un significado, no tener significado o tener varios significados. Como propiedades y relaciones no expresivas: ser analítica, ser antónima o ser contraria. Esto es lo que la teoría autónoma del sentido puede decir acerca del sentido de una oración independientemente de la evidencia referencial.

\section{La crítica de Cohen: un nuevo problema para el nuevo intensionalismo de Katz}

Como se ha argumentado, el intensionalismo que propone Katz evita la indeterminación del sentido. No obstante, un análisis de esta nueva propuesta parece arrojar resultados igual de problemáticos. Cohen afirma que si nos basamos en el nuevo intensionalismo que sostiene Katz, lo que determina la referencia de los términos de clases naturales son las definiciones reales y, por lo tanto, el sentido no es necesario. ¿Cómo explica la teoría autónoma del sentido la determinación de la referencia tipo de términos de clases naturales?

Determinar la referencia de un término es proporcionar condiciones necesarias y suficientes para pertenecer en cada mundo posible $(w)$ a la extensión de ese término. Se elige la referencia tipo de los términos de clases naturales porque, respecto a estos términos, el hecho de que algo caiga bajo su sentido no es en general suficiente para que sea miembro de su extensión-tipo. Los términos de clases naturales 
se distinguen por el tipo de cosas que denotan o pretenden denotar. Este grupo de objetos refleja distinciones reales en la naturaleza que tienen un papel explicativo y preciso en teorías científicas: «oro», «plata», «bronce», etcétera, son ejemplos de términos de clases naturales.

El análisis de Cohen ${ }^{1}$ parte del ejemplo de «aluminio» $\mathrm{y}$ «molibdeno». Supongamos que el sentido de ambos términos es el mismo. A partir del intensionalismo de Katz podríamos decir que a pesar de que tengan el mismo sentido, esas expresiones no son sinónimas — como en el caso de «dos»y «el número primo par»-, ya que sus propiedades intensionales son diferentes. La dificultad de determinar la referencia de estos dos términos es que sus sentidos no nos suministran suficiente información para elegir de alguna manera la referencia tipo. Para determinar la referencia de tales términos es necesaria la noción de mediación y la noción de correlatos referenciales. Debido a la autonomía de la teoría del sentido respecto a la teoría de la referencia, ahora la relación entre ellas es de mediación (MED): «el sentido media la referencia: el sentido no es suficiente para especificar las condiciones de la referencia [...] pero es necesario para especificar las condiciones para la referencia» (Katz, 2004: 47). En (MED) se impone la restricción de que los miembros de un término $t$ tengan el conjunto de propiedades que comprenden su sentido.

Además de (MED), los correlatos referenciales también son restricciones bajo las cuales se asignan colecciones de objetos del dominio a las expresiones del lenguaje. Los co-

\footnotetext{
${ }^{1}$ Cohen dirige su crítica sobre todo a las tesis que Katz desarrolla en su artículo «Analyticity, Necessity, and the Epistemology of Semantics» (1997). No obstante, creo que resulta enriquecedor complementar las tesis de Katz con lo expuesto en su libro Sense, Reference and Philosophy (2004). Esto no le quitará fuerza al argumento de Cohen.
} 
rrelatos referenciales nos dicen «cuándo caer bajo el sentido de una expresión es suficiente y cuándo no lo es; y [...] qué información se requiere para la membrecía en la extensióntipo, además de la de caer bajo su sentido» (Katz, 2004: 53-4). El correlato referencial que Katz propone para términos de clases naturales es el siguiente:

(RC3) Si el sentido (plenómico2) de una expresión no contiene la categoría clase natural, su extensión-tipo son las cosas del dominio que caen bajo ese sentido. Si el sentido (plenómico) de una expresión contiene la categoría clase natural, su referencia-tipo son las cosas del dominio que caen en ambas: en ese sentido y en la concepción de la naturaleza de la clase (2004: 54).

A partir de (RC3) una concepción explicativa de la naturaleza de la clase complementa la insuficiencia de los sentidos para determinar la referencia. En (RC3) se correlaciona «una propiedad particular del sentido, o una relación del sentido de las expresiones tipo, o de sus sentidos, con una propiedad o relación referencial» (Katz, 2004: 52). Debido a que los sentidos de las expresiones de las clases naturales son insuficientes para determinar sus extensiones tipo, su referencia puede describirse como referencia bajo descripción incompleta. De acuerdo con (RC3), una concepción explicativa de la naturaleza de la clase compensa la incompleción. Así, es un aspecto extralingüístico el que determina la referencia de un término de clase natural. Para Katz las concepciones explicativas son «concepciones científicas, concepciones del sentido común o proto-concepciones de los referentes, que

\footnotetext{
${ }^{2}$ Plenómicas: expresiones-tipo que son significativas y pueden tener extensión-tipo, como por ejemplo «soltero»y «número par».
} 
se asume capturan la esencia natural ejemplificada en ciertas instancias paradigmáticas de la aplicación del término» (2004: 54). De esta manera es necesario el sentido junto con el apoyo de conocimiento extralingüístico lo que determina la referencia de los términos de clases naturales.

Katz reconoce entonces que el conocimiento científico de los términos es la información que se requiere para distinguir las referencias de términos de clases naturales. «Katz ve esa (sintética) información como el suministro de una 'definición real' derivada no de algún aspecto de la estructura gramatical de la expresión (como lo son los sentidos), sino de una teoría científica» (Cohen, 2000: 127). Entonces, a partir de (RC3) el principio para determinar la referencia de un término de clase natural es que la referencia tipo de un término sean las cosas que caen tanto bajo su sentido como bajo la definición real del término.

De acuerdo con Cohen, el correlato referencial para términos de clases naturales de Katz es falso, pues parece que só-lo las definiciones reales pueden determinar correctamente la referencia. La estrategia de Katz es incluir correlatos referenciales a fin de complementar la insuficiencia de los sentidos para determinar la referencia. Para Cohen, «esta estrategia falla, excepto cuando, por casualidad, el sentido es abandonado y la referencia hace todo el trabajo (minando de ese modo el internalismo de Katz)» (2000: 128). El argumento de Cohen para demostrar lo anterior es como sigue:

(RC3) La extensión de un término de clase natural $(\mathrm{CN})$ en w es $\left(\mathrm{S}^{\wedge} \mathrm{R}\right)$.

i) $\mathrm{Si}(\mathrm{RC} 3)$ es verdadera, entonces todo objeto que pertenece a la extensión de $\mathrm{CN}$ pertenece también a (S).

ii) Hay objetos que pertenecen a la extensión de $\mathrm{CN}$, pero no pertenecen a $(S)$. 
iii) Si (RC3) es falsa, entonces el sentido de CN no es necesario ni suficiente para la pertenencia a la extensión de $\mathrm{CN}$.

iv) Si (iii) es verdadera, entonces la definición real de $\mathrm{CN}$ (más bien que $S \wedge R$ ) determina la referencia.

v) La definición real de $\mathrm{CN}$ determina la referencia de $\mathrm{CN}$.

Hay que recordar que una de las ausencias principales en la noción del sentido de Katz era la idea de que el sentido de una expresión determina la referencia de la expresión. El interés de Katz por una teoría autónoma del sentido era dar argumentos que mostraran que el sentido no debería determinar la referencia. Parte de su razón es la aceptación de los bien conocidos argumentos externistas de Putman y Kripke (ver Cohen, 2000: 117). En una teoría del sentido fundada en (D), mientras que las propiedades del sentido de una oración están determinadas por su sentido, las propiedades referenciales no lo están. Entonces Katz reconoce que la teoría autónoma del sentido por sí sola no puede determinar la referencia de una expresión e intenta complementar su teoría con la teoría de la referencia mediante una relación de mediación (MED).

La crítica de Cohen muestra que el correlato referencial para términos de clases naturales es falso. Según Katz, la extensión de un término de clase natural en $w$ es $\left(S^{\wedge} R\right)$, que es la intersección de (S): la clase de cosas que caen en el sentido de $\mathrm{CN}$ en $w$; y $(R)$ : la clase de cosas que caen bajo la definición real de $\mathrm{CN}$. Por lo que si este correlato referencial es correcto, entonces la extensión de $\mathrm{CN}$ es $(S \wedge R)$ y para pertenecer a $\left(S^{\wedge} R\right)$ hay que pertenecer a ambos. Sin embargo, hay casos en los que un objeto no pertenece al sentido descriptivo de un término pero sí a su extensión correcta. Supongamos que el sentido descriptivo de «aluminio» es la propiedad de ser ligero en peso, con un color blanco plateado, azulado, durable e 
inoxidable. Sin embargo, es concebible que existiera un metal con el mismo peso atómico del aluminio, pero que fuera café. Entonces habría un metal que cayera bajo la extensión «aluminio», pero que no satisfaría su sentido descriptivo (ser ligero en peso, con un color blanco plateado, azulado, durable e inoxidable). En tales casos el sentido no es necesario y entonces (RC3) es falso. La noción de sentido de Katz parte de la negación de que el sentido sea suficiente para determinar la referencia, y el argumento de Cohen muestra que tampoco es necesario; entonces parece que el sentido no tiene ningún papel en la teoría de la referencia. De ahí que Cohen concluya que la definición real, más bien que $(S \wedge R)$, determina la referencia de términos de clase natural.

Se planteó como objetivo de este texto revisar la noción del sentido de Katz y ver si las condiciones que proporciona son necesarias para determinar la referencia de un término de clase natural. Katz niega que caer en el sentido de un término $t$ sea suficiente para caer en la extensión de $t$. Sin embargo, acepta la tesis de que caer en el sentido de un término $t$ es necesario para caer en la extensión de $t$. Debido a que no es suficiente pero es necesario, Katz señala que el sentido media la referencia: el sentido es necesario a fin de especificar las condiciones para la referencia de las expresiones. Sin embargo, el argumento de Cohen basado principalmente en casos en los que un objeto no pertenece al sentido descriptivo de un término pero sí a su extensión correcta, contradice la tesis de Katz mostrando que hay casos en los que la extensión de un término sólo satisface su definición real, pero no su sentido descriptivo. Por lo que (RC3) es falsa y entonces el sentido tampoco es necesario. A favor de Katz y en contra del intensionalismo fregeano, el sentido no es suficiente para determinar la referencia. Sin embargo, el argumento de Cohen 
ha mostrado que tampoco es necesario, en contra del nuevo intensionalismo de Katz.

\section{Conclusiones}

A partir del rechazo de la noción fregeana del sentido, Katz logra bloquear el argumento de la determinación del sentido. El paso fundamental para ello fue postular una nueva definición del sentido en la que el sentido no determina la referencia, sino que determina propiedades y relaciones del sentido. Este paso llevó a Katz a cambiar la relación entre la teoría del sentido y la teoría de la referencia. A diferencia del intensionalismo fregeano, el nuevo intensionalismo de Katz -donde el sentido no es suficiente pero es necesario para determinar la referencia- mantiene una relación de mediación con la teoría de la referencia. Los correlatos referenciales le permiten a Katz explicar dicha relación y (RC3) fue el correlato referencial para los términos de clases naturales.

Si bien Katz logra con esto determinar el sentido, su intensionalismo se enfrenta, sin embargo, a un nuevo problema. Cohen ha mostrado que la estrategia de Katz para determinar la referencia de términos de clases naturales no demuestra que el sentido sea necesario para ello, sino todo lo contrario. Cohen se basa en casos en los que la extensión de un término únicamente satisface su definición real pero no su sentido descriptivo. Por lo tanto (RC3) es falso, ya que no es $(S \wedge R)$ sino sólo la definición real lo que determina la referencia. Entonces, a partir del análisis que se ha desarrollado, no parece haber argumentos para sostener que el sentido de un término $t$ es necesario para caer en la extensión de $t$. 


\section{BIBLIOGRAFÍA}

Cohen, J. (2000). Analyticity and Katz's New Intensionalism: or, If You Sever Sense form Reference, Analyticity is Cheap but Useless. En Philosophy and Phenomenological Research LXVI (1); 115-35.

Frege, G. (1998). Sobre sentido y referencia. En Ensayos de semántica y filosofía de la lógica (pp. 84-111), L. M. Villanueva (trad). Madrid: Tecnos.

Katz, J. (2004). Sense, Reference and Philosophy. New York: Oxford University Press. 


\section{RESUMEN}

En el presente artículo, el autor expone la teoría autónoma del sentido de Katz como una alternativa al intensionalismo fregeano. Según el autor, Katz argumenta que el intensionalismo fregeano padece del problema de la restricción demasiado débil, y esto lo lleva a la indeterminación del sentido. Un paso clave para librar tal problema es, según Katz, independizar la teoría del sentido de la teoría de la referencia. Sin embargo, a pesar de que con ello se logra rechazar el intensionalismo fregeano y así librar la indeterminación del sentido, la propuesta de Katz adquiere nuevos problemas para determinar la referencia de términos de clases naturales. Cohen señala que la única alternativa de Katz para explicar con éxito cómo se determina la referencia de estos términos es aceptar que las definiciones reales hacen todo el trabajo. Pero aceptar esto nos lleva a concluir que el sentido de un término $t$ no es necesario para caer en la extensión de t. Katz no puede aceptar tal conclusión puesto que su nuevo intensionalismo sostiene que el sentido es necesario pero no suficiente para la referencia. Por lo tanto, el autor argumenta que la estrategia de Katz fracasa al intentar determinar la referencia de términos de clases naturales.

Palabras clave: Katz, Frege, teoría autónoma del sentido e intensionalismo.

\section{ABSTRACT}

In this article, the author exposes the autonomous theory of sense of Katz as an alternative to fregean intensionalism. According to the author, Katz argues that fregean intensio- 
nalism has the problem of the too weak restriction and this leads him to indeterminacy of meaning. A key step to get rid this problem is, according to Katz, the independence between the theory of meaning from the theory of reference. However, despite the rejection of fregean intensionalism, Katz's proposal acquires new problems to determine the reference of natural kind terms. Cohen notes that the only alternative to explain successfully how to determine the reference of these terms is accepting that the actual definitions do all the work. But accepting this leads us to conclude that the meaning of a term $t$ does not necessary to fall in the range of $t$. Katz can't accept this conclusion, because he argues that sense is necessary but not sufficient for reference. Therefore, the author argues that the strategy of Katz fails when trying to determine the reference of natural kind terms.

Keywords: Katz, Frege, autonomous theory of sense and intensionalism. 